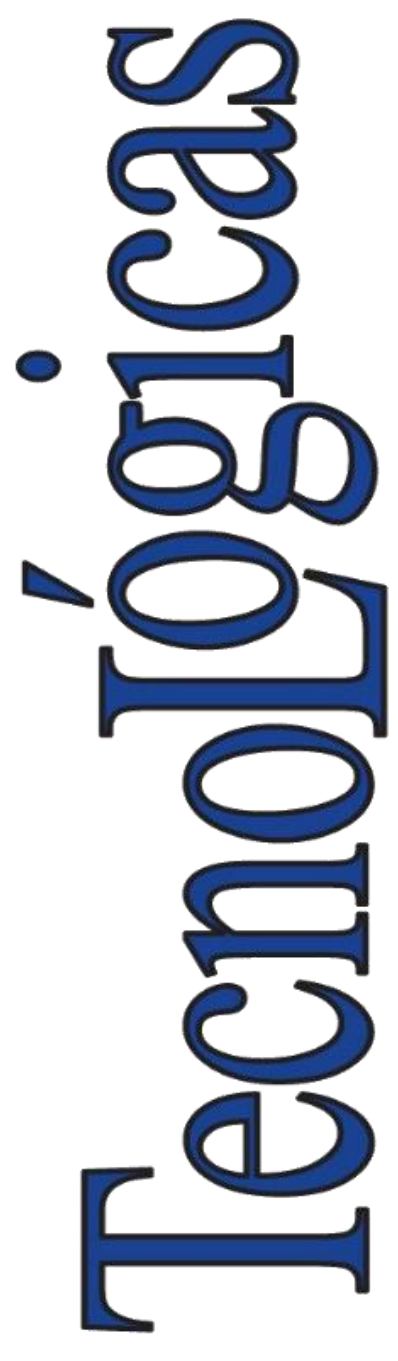

ISSN-p: 0123-7799

ISSN-e: $2256-5337$

Vol. 25, nro. 53, e2088, 2021

Recibido: 6 julio 2021 Aceptado: 25 enero 2022 Disponible: 01 marzo 2022

CInstituto Tecnológico Metropolitano

Este trabajo está licenciado bajo una Licencia Internacional

Creative Commons Atribución (CC BY-NC-SA)

\section{Metodologías empleadas para la producción de concreto permeable usando parcialmente materiales reciclados como agregados: una revisión literaria}

\author{
Methodologies to Produce Pervious Concrete Partially \\ Using Recycled Materials as Aggregates: A Literature \\ Review
}

\author{
(D) Jhon Emanuel Ayala-López ${ }^{1}$; \\ (iD) Elmer Gil-Ahumada ${ }^{2}$; \\ iD Rosario Dolores Cornejo-Ramos ${ }^{3}$; \\ (iD) Sócrates Pedro Muñoz-Pérez ${ }^{4}$

\begin{abstract}
${ }^{1}$ Universidad Señor de Sipán, Lambayeque - Perú, alopezjhon@crece.uss.edu.pe

2 Universidad Señor de Sipán, Lambayeque - Perú, gahumadaelmer@crece.uss.edu.pe

${ }^{3}$ Universidad Señor de Sipán, Lambayeque - Perú, cramosrosar@crece.uss.edu.pe

${ }^{4}$ Universidad Señor de Sipán, Lambayeque - Perú, msocrates@crece.uss.edu.pe
\end{abstract}

Cómo citar / How to cite

J. E. Ayala-López; E. Gil-Ahumada; R. D. Cornejo-Ramos; S. P. MuñozPérez, "Metodologías empleadas para la producción de concreto permeable usando parcialmente materiales reciclados como agregados: una revisión literaria”, TecnoLógicas, vol. 25, nro. 53, e2088, 2022. https://doi.org/10.22430/22565337.2088 


\section{Resumen}

El objetivo de la presente revisión fue recopilar y discutir literatura científica sobre metodologías empleadas para la producción y elaboración del concreto innovador "concreto permeable", como alternativa de diseño en pavimentos, con el remplazo parcial de agregado grueso por agregados reciclados, como látex de caucho al $5 \%$ y $8 \%$ por peso de cemento, ceniza volante entre el $0 \%$ y el $70 \%$ por volumen del cemento, y fibra de carbono al $0.27 \%$ y $0.4 \%$ por peso de agregado grueso. Esta revisión de literatura implicó seleccionar las publicaciones más relevantes, para los períodos del 2015 al 2021 en bases de datos indexadas Scopus, Scielo, ScienceDirect, y Latindex; enfocándose en múltiples parámetros como: "materiales residuales, coeficiente de permeabilidad, concreto permeable, grado de porosidad y ensayos de laboratorio para el concreto permeable". Los resultados muestran que la resistencia a la compresión varía entre $0.5 \mathrm{MPa}$ y $97.3 \mathrm{MPa}$, resistencia a la tracción entre 1.6 MPa y $5.29 \mathrm{MPa}$ y la permeabilidad entre $4.63 \mathrm{~mm} / \mathrm{s}$ y $10.2 \mathrm{~mm} / \mathrm{s}$, donde el porcentaje idóneo del uso de ceniza volante para la obtención óptima de concreto permeable es hasta el $20 \%$, que, excediendo este porcentaje, afecta negativamente sus propiedades mecánicas. En conclusión, el "concreto permeable" se sitúa dentro de los rangos establecidos según normativa: $17.5 \mathrm{MPa}, 2.6 \mathrm{MPa}, 6.1 \mathrm{~mm} / \mathrm{s}$, respectivamente; por ende, adquiere un valor sustancial, contribuyendo con la mejora del nivel microestructural y mayor durabilidad, siendo ventajoso y útil en los tiempos modernos.

\section{Palabras clave}

Concreto permeable, ensayos de laboratorio, materiales residuales, permeabilidad, porosidad.

\section{Abstract}

The objective of this literature review was to compile and discuss the scientific literature on methodologies used to produce and manufacture pervious concrete, an innovative design alternative in pavements. In pervious concrete, coarse aggregates are partially replaced with recycled aggregates; for instance, rubber latex at $5 \%$ and $8 \%$ by weight of cement, fly ash between $0 \%$ and $70 \%$ by volume of cement, and carbon fiber at $0.27 \%$ and $0.4 \%$ by weight of coarse aggregate. For this literature review, we selected the most relevant publications from 2015 to 2021 indexed in the Scopus, Scielo, ScienceDirect, and Latindex databases using keywords such as "residual materials", "coefficient of permeability", "pervious concrete", "degree of porosity", and "laboratory tests for pervious concrete". The results show that the compressive strength of this material varies between $0.5 \mathrm{MPa}$ and 97.3 $\mathrm{MPa}$; its tensile strength, between 1.6 MPa and 5.29 MPa; and its permeability, between $4.63 \mathrm{~mm} / \mathrm{s}$ and 10.2 $\mathrm{mm} / \mathrm{s}$. The desirable percentage of fly ash to optimize pervious concrete is up to $20 \%$; higher values negatively affect its mechanical properties. In conclusion, pervious concrete presents parameters within the ranges established by regulations: compressive strength, $17.5 \mathrm{MPa}$; tensile strength, 2.6 MPa; and permeability, $6.1 \mathrm{~mm} / \mathrm{s}$. Therefore, it has significant value because it contributes to microstructural level enhancement and greater durability, which makes it an advantageous and useful product in modern times.

\section{Keywords}

Pervious concrete, laboratory tests, residual materials, permeability, porosity. 


\section{INTRODUCCIÓN}

Actualmente, existen problemas causados por la escorrentía excesiva de aguas pluviales, así como la carencia de sistemas de drenaje en muchas ciudades, inundaciones repentinas en áreas urbanas, contaminación de mantos acuíferos, escases de agua, aumento de agua contaminada y afectaciones ambientales [1]. Es por ello por lo que el concreto permeable ha ganado buen interés en pavimentos debido a sus impactos ambientales positivos, para lo cual se desarrolla una extensa investigación con las diferentes estrategias para incrementar el rendimiento general del concreto permeable [2]. En años recientes, el concreto permeable es una opción sustentable para el diseño de pavimentos, cuya capa superficial permite el paso de agua de un punto a otro para así poder sacar provecho por medio de un procedimiento de recolección [3], siendo su primordial característica infiltrar el agua con la utilización de agregados reciclados (AR), como residuos generados en la construcción y cenizas volantes, logrando un aumento en la abrasión superficial [4]. Aunque no existe una metodología estandarizada para garantizar la medición correcta y precisa de dicha propiedad en condiciones de laboratorio [5], se lleva a cabo un programa experimental, enfocado a estudiar la influencia del volumen de la pasta sobre las propiedades finales (porosidad, densidad, velocidad de pulso ultrasónico, permeabilidad y resistencia a la compresión) del hormigón permeable [6], lo cual es muy utilizado en las industrias de la construcción de pavimentos debido a sus innumerables beneficios [7].

El concreto permeable de cemento Portland (CPCP) es revolucionario e innovador, tanto para la parte académica, como constructiva, por sus ventajas medioambientales, económicas y sociales [8]. Las diferentes propiedades del concreto permeable con agregados reciclados, tienden a la ley de distribución normal, entre ellas, la prueba de resistencia a la compresión, resistencia a la tracción, porosidad y coeficiente de permeabilidad y la absorción capilar de agua, generando una oposición entre la resistencia y la permeabilidad [9], estableciendo que la compactación juega un papel muy importante en las propiedades del concreto permeable [10]. Al igual que el mantenimiento regular puede mitigar las obstrucciones y prolongar la vida útil de los pavimentos de concreto permeable [11], entre una de sus características importantes prevalece la resistencia a la perforación, proporcionando dureza en los materiales altamente densificados, asemejándose a la resistencia de los cerámicos [12] utilizado en la industria para moldear cualquier forma a través de moldes rígidos llamados encofrados [13], siendo un medio eficaz para el control de la escorrentía de aguas pluviales y eliminación de contaminantes, formando una capa permeable superficial con una capa estructural, presentando una eficiencia de $83.8 \%$ a $95.2 \%$ [14]. Sabiendo que la compactación influye en las propiedades del concreto permeable y es evaluado mediante el procedimiento de proctor hummer con probetas cilíndricas y prismáticas; estableciendo un ábaco para determinar el número de golpes a compactar del concreto permeable relacionando el peso unitario y la porosidad [15], se concluye que las características más resaltantes del concreto permeable son: la porosidad, la composición y el proceso de compactación. Por ende, en lugar de depender de experimentos de prueba y error, sería mejor utilizar modelos numéricos avanzados, acelerando el proceso de producción, reducción del tiempo y costos [16].

Recomendado, para reducir el ruido en zonas urbanas, agregar a la mezcla cáscara de semilla de palma aceitera y cáscara de berberecho, produciendo pavimentos de concreto más limpios y permeables [17]. Cuando no cumple con los requisitos establecidos de dosificación y porcentaje es afectado por los agregados con una disminución de la resistencia a cargas monótonas y cíclicas, produciendo daños por humedad a mezcla del material granular ligado con cemento asfáltico, debido a la disminución de la temperatura de compactación [18]. La trabajabilidad del concreto reciclado reforzado con ladrillos de arcilla triturado, la resistencia 
a la tracción y la resistencia a la compresión del concreto reciclado es afectada significativamente por dichos materiales reciclados [19]. Al igual que la utilización de polímeros, destacando el látex de caucho de estireno-butadieno, emulsión de éster poliacrílico, agente impermeable de silicio orgánico que reduce la resistencia a la compresión y las cenizas volantes pueden mejorar este efecto adverso [20].

Este concreto permeable, al igual que el concreto convencional, es una mezcla de agregados de pasta de cemento, aditivos y poros de aire [21]. La sustitución de agregados naturales por $\mathrm{AR}$ es una alternativa sostenible; sin embargo, la composición influirá directamente en las propiedades mecánicas del concreto permeable [22], donde la porosidad, según el tipo del agregado, puede afectar la resistencia del concreto permeable, evaluado mediante la resistencia a la compresión con probetas de diferentes tamaños y formas [23]. La relación entre el tamaño del agregado, la estructura de poros, el espesor de la pasta cementosa tiene influencia en la resistencia a la compresión del concreto permeable [24].

Por lo tanto, el espesor del recubrimiento de la pasta en la superficie del agregado y el contenido de poros del agregado están influenciados en la compactación aplicada; no obstante, actualmente se desconoce el nivel de compactación requerido para lograr la densidad y la resistencia deseadas en campo [25], por lo cual se propone una estrategia numérica como alternativa que combina diferentes modelos para simular el proceso de compactación y predecir el rendimiento mecánico del concreto permeable, simulando la interacción mecánica de partícula-partícula en lo estructural, capaz de predecir los rendimientos experimentales de compresión y tracción, reduciendo así el número de pruebas [26].

Pero el concreto permeable debe ser diseñado evaluando sus propiedades mecánicas; por ende, la incorporación de nanosílice, acompañadas de cenizas volantes como un reemplazo parcial del cemento, mejora su resistencia a la compresión del concreto sin afectar la permeabilidad [27]. Agregando dióxido de titanio (TiO) mejora el nivel macro y microestructural, obteniendo mejor resistencia al biodeterioro y mayor durabilidad [28].

En este orden de ideas, el reemplazo de AR a la mezcla que sustituye al agregado grueso genera una mezcla con mayor porosidad obteniendo grandes ventajas para el drenaje de aliviaderos, pero también con deficiencias que afectan la resistencia mecánica, originando algunos riesgos en la estructura [29]. En efecto, la relación agua-cemento (A/C), reposición parcial de agregado grueso por agregado reciclado, intervienen en las propiedades, tanto físicas, como mecánicas, para generar el concreto permeable [30]. Por ello, los agregados gruesos son componentes importantes del concreto permeable [31]. También añadiendo fibras de acero al concreto tradicional se puede obtener concreto permeable de alta conductividad para aliviar el efecto de isla de calor urbano, tanto en condiciones secas, como húmedas [32].

Otro material que mejora la resistencia a la compresión, siendo una propiedad del concreto permeable, es el metacaolín, utilizado como complemento después de la sustitución de agregados naturales por agregados reciclados [33]. Los ladrillos de arcilla triturados son también una solución ideal para la gestión de residuos. que con un diseño apropiado podrían usarse en concreto permeable como base de pavimento [34]. Pero un estudio de los efectos del estearato de calcio, el oleato de sodio y el metilsiliconato de sodio, aplicados a la absorción de agua, hidratación, resistencia a la compresión, fluidez y periodo de fraguado en la pasta de cemento, dio como resultado que el estearato de calcio es el elemento más adecuado para la preparación de concreto permeable ligero [35]. Al sustituir el agregado natural por AR resultó en un incremento considerable en el coeficiente de permeabilidad, pero las propiedades mecánicas del concreto se ven influenciadas adversamente [36].

Por lo tanto, las metodologías de diseño consultadas buscan la obtención de un concreto con una permeabilidad al agua y resistencia a la congelación-descongelación adecuada para poder emplearse en pavimentos [37]. La mezcla con látex modificado logra una resistencia 
mucho mayor de durabilidad en la congelación-descongelación debido a que se mejora la unión de interfacial en la matriz cementosa [38]. No obstante, diseñar la mezcla de concreto permeable equilibrando la permeabilidad y resistencia, resulta un tanto complejo, ya que la estructura porosa permite que el concreto permeable alcance permeabilidad al agua, pero se reduce significativamente su resistencia [39], resaltando que el uso de cenizas volantes en un porcentaje mayor al $20 \%$ perjudica las propiedades mecánicas y físicas del concreto permeable [4]. Bajo esta situación, se desarrollaron concretos permeables sostenibles, utilizando residuos de la industria papelera, reduciendo el uso de cemento en aceras privadas y públicas [40]. Al igual que fibras y espumas, por su excelente coeficiente de absorción acústica a altas frecuencias son utilizadas ampliamente en el control pasivo del ruido, estos materiales requieren protección contra agentes ambientales y refuerzo estructural [41]. Al generar los residuos nace el interés de utilizar dichos materiales, como la cáscara de almendra de palma aceitera y la cáscara de berberecho, generando beneficios técnicosambientales, pero a su vez, disminuyendo la resistencia a la compresión [10].

Para obtener el concreto permeable se realizaron tres métodos: infiltración de anillo único de cabeza constante, infiltración de anillo único de cabeza descendente e infiltración de anillo doble [32]. Realizando un análisis de las características de la pasta de cemento, mezclando agregados con partículas de $1 / 4$ " a $3 / 8$ " y de $1 / 2$ " a $3 / 4$ ", con un curado adecuado de $20{ }^{\circ} \mathrm{C}$ durante 28 días [42], donde el concreto permeable mitiga el efecto del cambio climático, introduciendo una metodología de diseño modificada, basada en los estándares ACI 522R-10 y ACI 211.3R-02, que demuestra el aumento del $30 \%$ de la resistencia en sus capas superficiales y una disminución en la capacidad de infiltración del $40 \%$ [43]. Al reconocer y evaluar la obstrucción en concreto permeable con AR y agregados naturales, aprovechando que la permeabilidad varía, se obtuvo una variación de resultados de $17.5 \%$ y $19.6 \%$ de porosidad, para agregados reciclados y agregados naturales, respectivamente [44]. Entonces, el concreto permeable es capaz de soportar cargas de tráfico pesadas, estableciendo varios parámetros diseño con el fin de generar una metodología multicriterio, beneficiando, tanto a la ciudadanía como al medio ambiente; además, propone el uso de aditivos para ayudar a sus propiedades multifuncionales como fotocatálisis y reducción de ruido [45].

El interés de este manuscrito es utilizar los residuos en la producción de concreto permeable para obtener beneficios técnico-ambientales, utilizando cáscara de almendra de palma aceitera, cáscara de berberecho y ladrillos de arcilla triturado incrementando el coeficiente de permeabilidad, sin dejar al lado las cenizas volantes y el dióxido de titanio que incrementan la resistencia a la compresión sin afectar la propiedad de permeabilidad del concreto, donde la escorrentía excesiva de aguas pluviales es un problema para el pavimento, siendo una alternativa sostenible el uso de agregados grueso reciclados con una porosidad varía de $15 \%$ a $35 \%$ según el tipo y tamaño, para el cual la eficacia del concreto permeable se evalúa de acuerdo a la permeabilidad, estabilidad y resistencia mecánica; por lo tanto, la relación $\mathrm{A} / \mathrm{C}$ y reposición de agregado grueso interviene sobre las propiedades del concreto permeable, tanto físicas, como mecánicas. Siendo un desafío crítico para el ingeniero equilibrar la permeabilidad y resistencia del concreto, generan oportunidades en el sector de la construcción, y mayor expansión e interés en la aplicación metodológica en la producción para concreto permeable. 


\section{METODOLOGíA}

Las propuestas metodológicas para la elaboración y revisión se orientaron en la literatura más prestigiosa y de acuerdo con el tema de estudio, lo cual implicó seleccionar las publicaciones más relevantes para los períodos del 2015 al 2021. Para los criterios de búsqueda se elaboró una bitácora considerando cuatro de las principales bases de datos indexadas, como Scopus, Scielo, ScienceDirect, y Latindex, donde las palabras clave usadas son: materiales residuales u agregados reciclados para el concreto permeable, coeficiente de permeabilidad del concreto, concreto permeable, concreto poroso, dosificación y usos del concreto permeable, grado de porosidad del concreto, ensayos de laboratorio para el concreto permeable y propiedades del concreto permeable, donde clasificaron un total de 77 artículos.

Se formaron dos matrices; en la primera, se analizaron los criterios de búsqueda y selección de artículos, en la cual se observa: palabras clave, total de documentos encontrados según base de datos, los años de búsqueda según base de datos, la cantidad de documentos filtrados según año de búsqueda, aplicación de ecuación de búsqueda según áreas (ingeniería, artículo científicos y artículos de revisión con acceso abierto), obteniendo mejores resultados con los filtros y así, finalmente, obtener los artículos esenciales para esta investigación. Para mayor detalle, en la Tabla 1 se muestra el motor de búsqueda y elección de artículos usados en la investigación.

Tabla 1. Motor de búsqueda y elección de artículos usados en la investigación Fuente: elaboración propia.

\begin{tabular}{|c|c|c|c|c|c|c|c|}
\hline Base de datos & Palabra clave & $\begin{array}{c}\text { Total de } \\
\text { documentos }\end{array}$ & $\begin{array}{c}\text { Año de } \\
\text { búsqueda }\end{array}$ & $\begin{array}{l}\text { Cantidad de } \\
\text { documentos }\end{array}$ & $\begin{array}{c}\text { Ecuación de la } \\
\text { búsqueda }\end{array}$ & $\begin{array}{l}\text { Resultado } \\
\text { final con } \\
\text { filtro }\end{array}$ & $\begin{array}{l}\text { Artículos } \\
\text { elegidos }\end{array}$ \\
\hline \multirow{7}{*}{ Scopus } & Pervious concrete & 1274 & $2015-2021$ & 835 & $\begin{array}{c}\text { All Open Access + } \\
\text { Engineering }+ \\
\text { Article. }\end{array}$ & 71 & 12 \\
\hline & Porpous concrete & 5245 & $2016-2021$ & 2070 & $\begin{array}{c}\text { All Open Access + } \\
\text { Engineering }+ \\
\text { Article. }\end{array}$ & 211 & 20 \\
\hline & $\begin{array}{l}\text { Recycled } \\
\text { aggregates for } \\
\text { concrete }\end{array}$ & 8020 & $2015-2021$ & 5329 & $\begin{array}{c}\text { All Open Access + } \\
\text { Engineering + } \\
\text { Article. }\end{array}$ & 595 & 8 \\
\hline & $\begin{array}{l}\text { Coefficient of } \\
\text { permeability of } \\
\text { concrete }\end{array}$ & 1530 & $2015-2021$ & 751 & $\begin{array}{c}\text { All Open Access + } \\
\text { Engineering }+ \\
\text { Article. }\end{array}$ & 86 & 4 \\
\hline & $\begin{array}{l}\text { Dosage and uses } \\
\text { of pervious } \\
\text { concrete }\end{array}$ & 8 & $2017-2021$ & 7 & $\begin{array}{c}\text { Engineering + } \\
\text { Article. }\end{array}$ & 3 & 0 \\
\hline & $\begin{array}{l}\text { Degree of porosity } \\
\text { of concrete }\end{array}$ & 567 & $2015-2021$ & 276 & $\begin{array}{c}\text { All Open Access + } \\
\text { Engineering }+ \\
\text { Article. }\end{array}$ & 37 & 1 \\
\hline & $\begin{array}{l}\text { Tests for pervious } \\
\text { concrete }\end{array}$ & 435 & $2015-2021$ & 303 & $\begin{array}{c}\text { All Open Access + } \\
\text { Engineering }+ \\
\text { Article. }\end{array}$ & 29 & 1 \\
\hline \multirow{2}{*}{ Scielo } & Pervious concrete & 14 & $2015-2021$ & 13 & $\begin{array}{l}\text { Enginnering + } \\
\text { Civil + Article. }\end{array}$ & 10 & 3 \\
\hline & Porous concrete & 14 & $2016-2021$ & 5 & $\begin{array}{l}\text { Enginnering + } \\
\text { Civil+ Article. }\end{array}$ & 4 & 1 \\
\hline
\end{tabular}




\begin{tabular}{|c|c|c|c|c|c|c|c|}
\hline & $\begin{array}{l}\text { Recycled } \\
\text { aggregates for } \\
\text { concrete }\end{array}$ & 72 & $2015-2021$ & 45 & $\begin{array}{l}\text { Enginnering + } \\
\text { Civil+ Article. }\end{array}$ & 43 & 6 \\
\hline & $\begin{array}{l}\text { Coefficient of } \\
\text { permeability of } \\
\text { concrete }\end{array}$ & 9 & $2017-2021$ & 6 & $\begin{array}{l}\text { Enginnering + } \\
\text { Civil+ Article }\end{array}$ & 4 & 1 \\
\hline & $\begin{array}{l}\text { Dosage and uses } \\
\text { of pervious } \\
\text { concrete }\end{array}$ & --- & $-\cdots$ & $--\cdot$ & --- & $-\cdots$ & $-\cdots$ \\
\hline & $\begin{array}{l}\text { Degree of porosity } \\
\text { of concrete }\end{array}$ & $\cdots$ & $\cdots$ & $\cdots$ & $\cdots$ & $\cdots$ & $\cdots$ \\
\hline & $\begin{array}{l}\text { Tests for pervious } \\
\text { concrete }\end{array}$ & 6 & $2018-2021$ & 6 & $\begin{array}{l}\text { Enginnering + } \\
\text { Civil+ Article }\end{array}$ & 4 & 1 \\
\hline \multirow{8}{*}{ ScienceDirect } & Pervious concrete & 2459 & $2015-2021$ & 1425 & $\begin{array}{c}\text { Review articles + } \\
\text { Engineering. }\end{array}$ & 53 & 9 \\
\hline & Porous concrete & 43178 & $2017-2020$ & 12259 & $\begin{array}{l}\text { Review articles + } \\
\text { Engineering. }\end{array}$ & 409 & 9 \\
\hline & $\begin{array}{l}\text { Recycled } \\
\text { aggregates for } \\
\text { concrete }\end{array}$ & 19235 & $2015-2020$ & 9462 & $\begin{array}{l}\text { Review articles + } \\
\text { Engineering. }\end{array}$ & 354 & --- \\
\hline & $\begin{array}{l}\text { Coefficient of } \\
\text { permeability of } \\
\text { concrete }\end{array}$ & 21496 & $2016-2021$ & 10338 & $\begin{array}{l}\text { Review articles + } \\
\text { Engineering. }\end{array}$ & 397 & 1 \\
\hline & $\begin{array}{l}\text { Dosage and uses } \\
\text { of pervious } \\
\text { concrete }\end{array}$ & 290 & $2016-2021$ & 227 & $\begin{array}{l}\text { Review articles + } \\
\text { Engineering. }\end{array}$ & 22 & $-\cdots$ \\
\hline & $\begin{array}{l}\text { Degree of porosity } \\
\text { of concrete }\end{array}$ & 24369 & $2016-2021$ & 12586 & $\begin{array}{l}\text { Review articles + } \\
\text { Engineering. }\end{array}$ & 491 & $\cdots$ \\
\hline & $\begin{array}{l}\text { Properties of } \\
\text { permeable } \\
\text { concrete }\end{array}$ & 362324 & $2015-2021$ & 179884 & $\begin{array}{l}\text { Review articles + } \\
\text { Engineering. }\end{array}$ & 635 & 3 \\
\hline & $\begin{array}{l}\text { Tests for pervious } \\
\text { concrete }\end{array}$ & 1983 & $2015-2020$ & 1206 & $\begin{array}{l}\text { Review articles + } \\
\text { Engineering. }\end{array}$ & 51 & --- \\
\hline \multirow[b]{2}{*}{ Latindex } & Revista en línea & 11695 & $\begin{array}{c}\text { Ciencias de } \\
\text { la Ingeniería } \\
\text { + Revista de } \\
\text { Investigació } \\
\text { n Científica } \\
\text { + Acceso } \\
\text { abierto }\end{array}$ & \multicolumn{2}{|r|}{$\begin{array}{c}\text { Ciencias de la } \\
\text { Ingeniería + } \\
\text { Revista de } \\
\text { Investigación } \\
\text { Científica + } \\
\text { Acceso abierto + } \\
\text { Tecnología. }\end{array}$} & 97 & 3 \\
\hline & Revista en línea & 11695 & $\begin{array}{c}\text { Ciencias de } \\
\text { la Ingeniería } \\
\text { + Revista de } \\
\text { Investigació } \\
\text { n Científica } \\
\text { + Acceso } \\
\text { abierto }\end{array}$ & 250 & $\begin{array}{c}\text { Ciencias de la } \\
\text { Ingeniería + } \\
\text { Revista de } \\
\text { Investigación } \\
\text { Científica + } \\
\text { Acceso abierto + } \\
\text { Ingeniería de } \\
\text { materiales. }\end{array}$ & 20 & 3 \\
\hline Total & & 515918 & & 237533 & & 3626 & 83 \\
\hline
\end{tabular}


Luego de pasar por filtros en la segunda matriz se detalla la cantidad de artículos extraídos por año de publicación de las diferentes bases de datos indexadas, conformando un subtotal por año 10 en el 2021, 21 en el 2020, 16 en el 2019, 8 en el 2018, 4 en el 2017, 9 en el 2016 y 4 en el 2015, para un total de 72 artículos seleccionados. Para mayor detalle, en la Tabla 2 se muestra los artículos distribuidos para cada base de datos indexados y publicación por año.

Tabla 2. Artículos distribuidos para cada base de datos indexados y publicación por años Fuente: elaboración propia.

\begin{tabular}{|c|c|c|c|c|c|}
\hline \multirow{2}{*}{ Año } & \multicolumn{4}{|c|}{ Base de datos } & \multirow{2}{*}{ Total } \\
\hline & Scielo & Scopus & Latindex & SciencieDirect & \\
\hline 2015 & 1 & 1 & 1 & 1 & 4 \\
\hline 2016 & 1 & 3 & & 5 & 9 \\
\hline 2017 & & 3 & 1 & & 4 \\
\hline 2018 & 1 & 4 & 1 & 2 & 8 \\
\hline 2019 & 2 & 10 & 2 & 3 & 17 \\
\hline 2020 & 5 & 15 & 1 & 7 & 28 \\
\hline 2021 & & 10 & & 3 & 13 \\
\hline Total & 10 & 46 & 6 & 21 & 83 \\
\hline
\end{tabular}

\section{RESULTADOS Y DISCUSIÓN}

\subsection{Métodos}

La investigación se basa en el aprovechamiento de agregados reciclados para el estudio de concreto permeable con la finalidad de realizar diferentes ensayos a base de agregados reciclados (AR) [17]. Para esto se investigó los parámetros de porosidad y coeficiente de permeabilidad [5], donde la estructura de concretó permeable va a depender de las variables de la mezcla, como las dosificaciones de cemento, agua y agregados [46]. Por lo tanto, para el desarrollo de esta investigación es importante tener en cuenta el aprovechamiento de AR, así como la trituración y el tamizaje para ser utilizado como material de AR [47].

Entonces, con grava natural de 1/4" a 3/8", cemento Portland y látex de caucho de estireno butadieno (SBR), se produjo concreto permeable con dosificación de cemento-agregado (1: 4.5) con $0 \%$ de polímeros, utilizando $400 \mathrm{~kg}$ de cemento por metro cubico de concreto, $1800 \mathrm{~kg}$ agregado grueso por metro cubico de concreto, con relación $\mathrm{A} / \mathrm{C}$ entre 0.30 y 0.35 , también dos porcentajes de SBR de $5 \%$ y $8 \%$ por peso de cemento [48]. Asimismo, se incorporó fibra de carbono curado parcialmente al concreto permeable, utilizado como agregado grueso en diferentes proporciones de $4.15 \mathrm{~kg} / \mathrm{m}^{3}$ y $5.9 \mathrm{~kg} / \mathrm{m}^{3}$ de concreto, pero en porcentaje representa, tanto 0.27 de volumen, como 0.4 volumen [49]. Basándose en un diseño experimental ortogonal, con relación $\mathrm{A} / \mathrm{C}$ de 0.37 , porosidad del $20 \%$, contenido de volumen de $0.16 \%$, se obtiene resistencia a la compresión superior a $20 \mathrm{MPa}$, resistencia a la flexión mayor a 2.5 MPa, alta permeabilidad al agua con coeficiente de permeabilidad mayor a $20 \mathrm{~mm} / \mathrm{s}$ [37].

Mostrando que las casaras de conchas marinas trituradas influyen en la durabilidad del concreto permeable con el reemplazando del $60 \%$ de la masa de los agregados naturales [50]; al igual que ceniza volante al $0 \%, 10 \%, 20 \%, 30 \%, 40 \%, 50 \%, 60 \%$ y $70 \%$ como una sustitución parcial del cemento por volumen y nanosílice al $0 \%, 1 \%, 2 \%$ y $3 \%$ como aditivos [27]. En otro caso, utilizaron polvo de diatomita y zeolita; primero usaron dihidróxido de 
magnesio para modificar la diatomita; luego, la diatomita modificada y el polvo de zeolita se adoptaron de manera innovadora como aglutinantes para preparar concreto permeable; la diatomita modificada al $10 \%$, y el polvo de zeolita al $3 \%$, exhiben la mayor correlación con las propiedades del concreto permeable modificado [51]. Además, seleccionando las diferentes porosidades desde el $8 \%$ al $30 \%$, escoria de hierro como sustitución de agregado grueso para generar concreto permeable, con una relación $\mathrm{A} / \mathrm{C}$ de $0.23 \%$ y humo de sílice-cemento $6 \%$ [31]. El uso de escoria mejora el índice de reflectancia solar con agregado de piedra caliza sin afectar negativamente la resistencia a la compresión o la permeabilidad [52].

En la Figura 1 se presentan los diferentes tipos de agregados reciclados utilizados para la elaboración del concreto permeable según la relación $\mathrm{A} / \mathrm{C}$ desde 0.23 hasta 0.40 y los porcentajes de sustitución del agregado grueso por agregados reciclados desde el $0 \%$ hasta el $70 \%$, donde los resultados difieren en cada investigación, por ejemplo: [53] utilizaron tres diferentes tipos de grava para tamaño máximo 3/4" y para tamaño mínimo 3/8", según menciona la Norma ACI 522R-06, y así poder mejorar la trabajabilidad de la mezcla dando mejor resultado con aditivo superplastificante-retardante. Por su parte, [54] tuvieron que utilizar 5 AR y 1 agregado natural para poder generar concreto permeable; dentro de los agregados reciclados destacan los ladrillos de arcilla triturado al $10 \%, 25 \%, 50 \%$ y $100 \%$ del total para la dosificación cemento-agregado (1:5) y en la relación A/C 0.30. De igual forma, [55] analizaron ocho muestras de concreto permeable con la sustitución de arena al $0 \%$ y $8 \%$ por peso de agregado grueso, incorporando inclusores de aire $\left(0 \mathrm{y} 333 \mathrm{ml} / \mathrm{m}^{3}\right)$, relaciones $\mathrm{A} / \mathrm{C}$ tanto de 0.30 como 0.40, y con agregados gruesos desde 1/4" a 3/4". Entretanto, [10] utilizaron cemento Portland tipo I, agregado grueso natural (piedra caliza triturada de 1/4"), y una gravedad específica de $2.7 \mathrm{~kg} / \mathrm{m}^{3}$. Asimismo, [56] utilizaron cuatro diferentes tipos de agregados: ladrillos de cerámicos triturados, escoria de horno eléctrico, basalto y residuos de concreto triturado. En este caso se obtuvo un contenido de cemento de $420 \mathrm{~kg} / \mathrm{m}^{3}$ y agregado grueso $1369.20 \mathrm{~kg} / \mathrm{m}^{3}$. Para la relación A/C se optó 0.34 para todos. Por otro lado, [57] tuvieron que elegir tres tipos de mezclas para concreto permeable con relaciones $\mathrm{A} / \mathrm{C}$ de $0.28,0.30 \mathrm{y}$ 0.34 y varios niveles de sustitución de AR: $100 \%$ (AR1) ladrillo cerámico triturado y $0 \%$ (AR2) concreto triturado, $75 \%$ AR1 - $25 \%$ AR2, $50 \%$ AR1 - $50 \%$ AR2, $25 \%$ RA1 - $75 \%$ RA2 y $0 \% \mathrm{AR} 1$ - $100 \% \mathrm{AR} 2$; además, se incorporó policarboxílico modificado como aditivo al $0.3 \%$ por peso del cemento. Desde otro ángulo, [58] fabricaron diez tipos de concretos permeables, sustituyendo los agregados naturales con cenizas volantes adheridas en frío en relación con $\mathrm{A} / \mathrm{C}$ de 0.27 y 0.32 , alcanzando así un valor máximo en el coeficiente de permeabilidad de $10.27 \mathrm{~mm} / \mathrm{s}$ para una relación A/C de 0.32. Igualmente, [59] disminuyeron el uso del cemento en pavimentos, remplazando parcialmente el cemento Portland por metacaolín, donde la sustitución fue del $5 \%$ de cemento, demostrando mayor resistencia a la tracción indirecta y permeabilidad. Además, [38] utilizaron como agregado grueso la piedra caliza para lograr una eficiente resistencia, al igual que una estabilidad de gradación agregando una pequeña cantidad de arena de río, también se agregaron una cierta cantidad de emulsión de etilenoacetato de vinilo (EVA) y fibra polipropileno monofilamento.

Pero también se realizó un estudio con la probabilidad de poder usar un pavimento de concreto permeable en la clarificación de aguas residuales; se utilizaron dos tamaños de grava G1 agregado entre 1/4"- 3/8, G2 agregado entre 3/8"- 3/4" con tres relaciones A/C de 0.28, 0.30 y 0.33 , donde la resistencia máxima a la compresión fue de $17.75 \mathrm{MPa}$ con la grava de G1 y la relación A/C más alta [60]. 


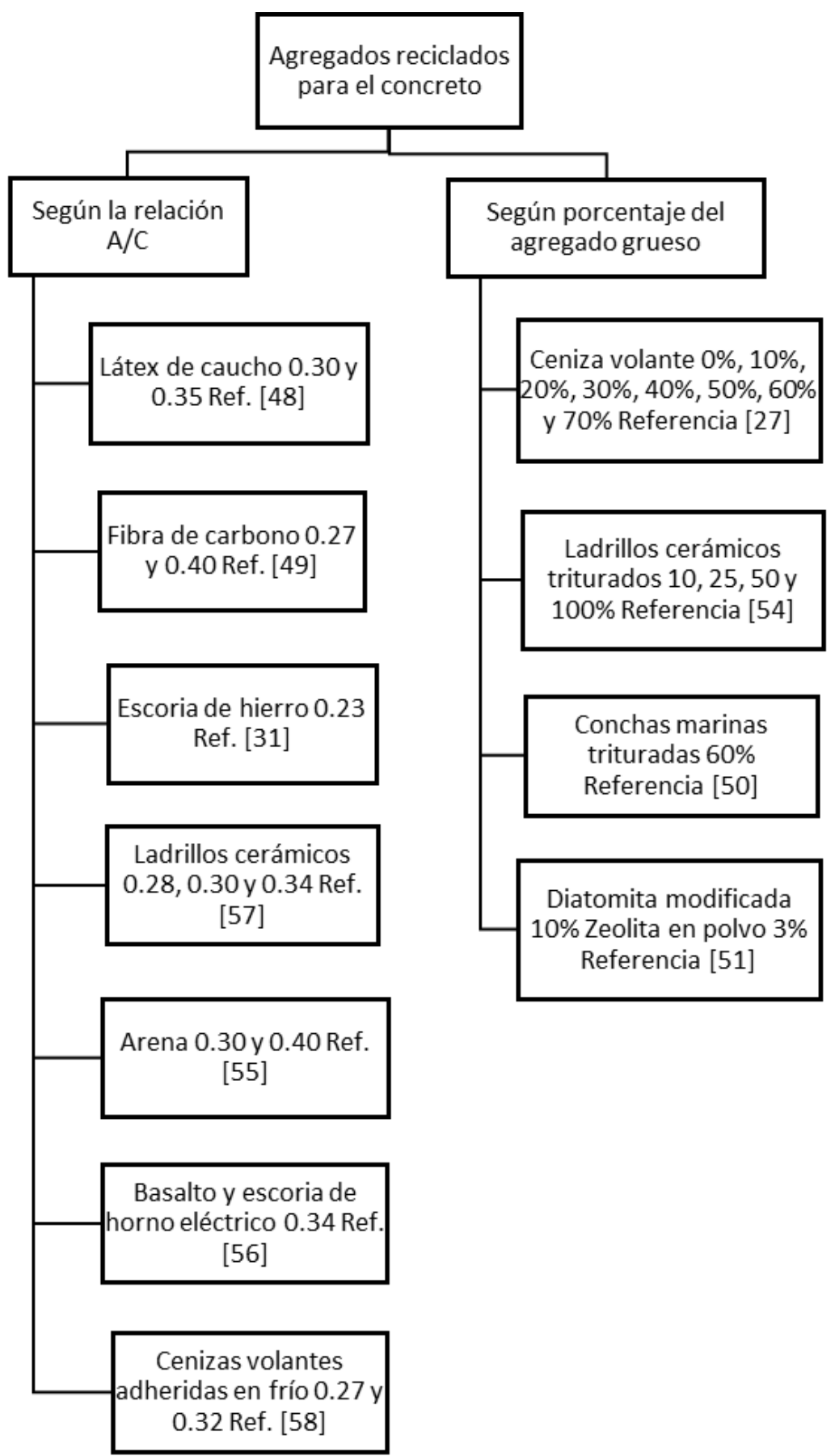

Figura 1. Agregados reciclados utilizados para la elaboración del concreto permeable según la relación A/C y porcentaje de sustitución del agregado grueso. Fuente: elaboración propia.

\subsection{Métodos y ensayos para el concreto permeable}

La determinación precisa de la permeabilidad en campo es una base importante para evaluar el desempeño y mantenimiento del pavimento de concreto permeable, precisando tres métodos de prueba de permeabilidad de campo: infiltración de anillo único con cabezal constante (CH-SRI), infiltración de anillo único con cabezal descendente (FH-SRI) e infiltración de anillo doble (DRI) [61]. El infiltrómetro con $100 \mathrm{~mm}$ de diámetro en anillo 
simple, por el método de Best-Slope, BeerKan y Best-Intercept, al igual que otros como la ecuación de Genutchen, Corey e BrooKs, y cuyos efectos de infiltración son tridimensionales medidos en campo [62]. Tambien se empleó el infiltrómetro, pero de anillo único con $300 \mathrm{~mm}$ de diámetro referenciado según el ASTM C1701, pero influenciado por el cambio de clima, utilizando agregado grueso y fino para verificar la variación de infiltración, obteniendo mejores resultados el agregado grueso de 1/4" y 3/8" [63], donde la recomendación del ASTM C1701 establece que hay una prueba para medir la tasa de infiltración, siendo este el coeficiente de permeabilidad usado para estimar el comportamiento de permeabilidad en el análisis del pavimento [64]. Además, los ensayos de porosidad y permeabilidad se pueden obtener con el método propuesto de Nguyen; también utilizaron el método de caída de la cabeza para medir la permeabilidad al agua, así como para el cálculo de coeficiente de permeabilidad, la ley de Darcy [50]. Otro método utilizado fue el de Corelok (ASTM D7063) para pavimentos permeables, sumergido por 30 minutos a una temperatura de $25{ }^{\circ} \mathrm{C}$, utilizando asfalto permeable, concreto permeable y material de pavimento de caucho permeable [65].

Por lo tanto, para evaluar la resistencia a la comprensión utilizaron probetas de $100 \mathrm{~mm}$ y $200 \mathrm{~mm}$ en un tiempo correlativo de 3 y 5 minutos; para la prueba a la flexión se utilizaron prismas de $200 \mathrm{~mm}, 400 \mathrm{~mm}$ y $70 \mathrm{~mm}$, el varillado se dio de 25 veces con varilla de apisonamiento de $10 \mathrm{~mm}$ de diámetro, todas las muestras fueron desmoldadas a la edad de 24 horas [47]. Se probaron un total de 23 muestras, las cuales fueron cargadas a una tasa de $0.0005 \mathrm{~mm} / \mathrm{s}$, alcanzando una carga máxima en dos minutos, controlado por un compactador automático [66], donde la cantidad de cemento utilizada no solo tiende a afectar el espesor del recubrimiento, sino también a la porosidad y a las propiedades mecánicas del concreto [67]. Al igual que para evaluar la conductividad hidráulica en el concreto permeable, hay que tener en cuenta el diseño de una celda permeable con tubo acrílico de trescientos mm de largo, y con ciento diez mm de diámetro interno, con una muestra encerrada que no absorbe agua [68]. Otro es la prueba de lixiviación, que es la disolución del hidróxido de calcio de la pasta de cemento y la liberación del catión de calcio e ion de hidróxido, aumentando la porosidad [54]. Donde la curva de presión de compactación se evalúa con una carga máxima de $10 \mathrm{kN} \mathrm{y}$ $1 \%$ de presión que opera en modo de desplazamiento a una velocidad de $3.33 * 10-\mathrm{M} / \mathrm{S}$, utilizaron cilindros de compactación de $95 \mathrm{~mm}$ de diámetro y una altura de $65 \mathrm{~mm}$, añadiendo $1 \%$ de retardante en peso de cemento con el fin de disminuir las variaciones en las propiedades del hormigón [69].

El método de diseño para obtener la porosidad deseada es mediante la incorporación de un aditivo modificador de la reología, mostrando mejorías en la densificación y fluidez de la mezcla, donde la disminución de la relación A/C no influye en la resistencia a la compresión, pero incrementa la resistencia a la tracción por flexión [70]. Asimismo, se aplicó el método ultrasónico para el concreto permeable como ensayo no destructivo, donde la velocidad del pulso ultrasónico oscila entre $3642 \mathrm{~m} / \mathrm{s}$ a $4262 \mathrm{~m} / \mathrm{s}$, con una reducción de la porosidad al $12 \%$; es decir que la velocidad de pulso ultrasónico se eleva a medida que la porosidad disminuye, afectando la permeabilidad del concreto. Además, las propiedades mecánicas (resistencias a la compresión y a la tracción) son inversamente proporcionales a la velocidad del pulso ultrasónico [71].

En la Figura 2 se muestra los diferentes métodos utilizados en la producción de concreto permeable para determinar la permeabilidad y la variedad de ensayos realizados al concreto permeable con sus valores respectivos. 


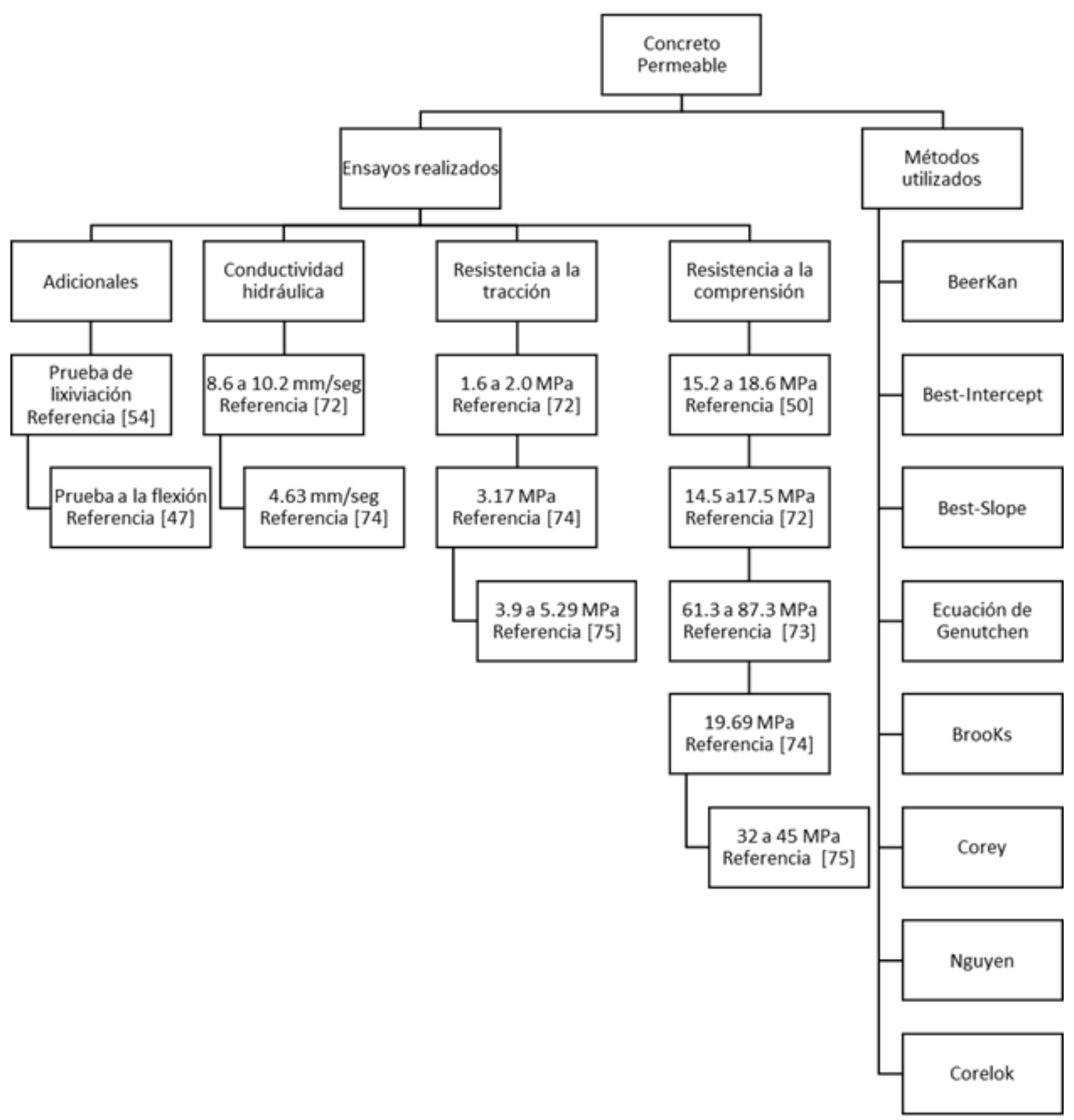

Figura 2. Métodos para determinar la permeabilidad y sus ensayos al concreto permeable Fuente: elaboración propia.

Los resultados de los ensayos difieren en cada investigación, por ejemplo: [50] mencionan que la resistencia a la comprensión del concreto permeable varió entre 15.2 $\mathrm{MPa}$ a 18.6 MPa, disminuyendo desde un $13.2 \%$ al $29.2 \%$ de la resistencia a la tracción. Entre tanto, [72] mencionan que la resistencia a la compresión varia de 14.5 a $17.5 \mathrm{MPa}$, la resistencia a la tracción de 1.6 MPa a 2.0 MPa y la conductividad hidráulica desde $8.6 \mathrm{~mm} / \mathrm{s}$ hasta $10.2 \mathrm{~mm} / \mathrm{s}$. Mientras tanto, [73] mencionan que la resistencia a la compresión a los siete días permanece entre 61.3 MPa a 87.3 MPa, 53.37\% de superplastificantes, y contenido de agentes viscosos, $9.65 \%$. Por lo tanto, [74] mencionan que la resistencia a la compresión fue de $19.69 \mathrm{MPa}$ a los 28 días, adicionándole polipropileno al $0.05 \%$, el módulo de rotura o resistencia a la 
tracción de $3.17 \mathrm{MPa}$ y permeabilidad de $4.63 \mathrm{~mm} / \mathrm{s}$. Por su parte, [75] determinaron que, tanto las características hidráulicas como mecánicas del concreto, utilizando cemento Portland, generan una resistencia a la compresión desde $32 \mathrm{MPa}$ hasta $45 \mathrm{MPa}$ y una resistencia a la flexión que varía desde 3.9 MPa hasta 5.29 MPa durante 28 días sin dejar de lado el tema de curado y las ventajas del uso de concreto permeable. En las aplicaciones del concreto permeable las ventajas son:

Ambiental: el uso del concreto permeable resuelve eficazmente el problema de la contaminación del agua siendo un material de construcción ecológico importante, que se ajusta al concepto de "ciudad esponja" [76]. También el uso de AGR, como son los residuos generados por la construcción y demolición, son una solución sostenible con múltiples beneficios ambientales [77]. Mediante pruebas hidromecánicas, plantea el uso de conchas marinas provocando un sistema interconectado de poros, accediendo así el libre tránsito del agua y sosteniendo un equilibrio de su resistencia mecánica [78]. También propusieron bajar la capacidad de escorrentía de un piso entre el suelo y el cemento, tuvieron que evaluar el coeficiente de flujo, volumen de escorrentía, volumen precipitado, destacando que el pavimento suelo-bloques de cemento va a ser semipermeable [79].

Las aplicaciones a gran escala de pavimentos permeables podrían ayudar a mitigar los impactos de las "islas de calor urbano" [80]. El concreto permeable garantiza un buen comportamiento estructural, funcional y medioambiental en un barrio urbano, donde el $80 \%$ de la superficie es impermeable, aportando un valor arquitectónico y estético para el medio ambiente [81].

Económico: se sabe que la cantidad de cemento utilizado en la producción de concreto permeable es menor que la de concreto común, lo que reduce el consumo de energía y la emisión de $\mathrm{CO}_{2}$ [82]. Así, como con la adición de cenizas volantes, muestra un desempeño más alto, considerando el equilibrio de múltiples criterios en propiedades de ingeniería, ahorro de costos, energía y reducción de gases de efecto invernadero [83].

\section{CONCLUSIONES}

A partir de la información recabada durante esta investigación, se plasma el estudio de diferentes sustituciones de materiales a base de agregados reciclados, para la elaboración de un concreto permeable, concluyendo lo siguiente:

La utilización de algunas metodologías para la producción de concreto permeable, a base de agregados reciclados, con relación de $\mathrm{A} / \mathrm{C}$ desde 0.23 a 0.40 , generan un buen comportamiento de la mezcla.

La relación $\mathrm{A} / \mathrm{C}$, y el uso de agregados reciclados parcialmente, influyen en el aumento del contenido de vacíos y coeficiente de permeabilidad del concreto, limitando su utilización en pavimentos de bajo tránsito, veredas y ciclovías.

Asimismo, la relación $\mathrm{A} / \mathrm{C}$ superior a 0.50 en combinación con agregado reciclados de tamaño máximo nominal de 3/8", genera una obstrucción de la estructura porosa interna del concreto, perdiendo su funcionalidad.

En cuanto a la elaboración del concreto permeable se sustituye parcialmente el agregado grueso, por diferentes agregados reciclados como: látex de caucho, fibras de carbono, escoria de hierro, cenizas volantes adheridas en frío y ladrillos de arcilla triturado.

El incremento de la granulometría del agregado aumenta la porosidad de la mezcla, pero genera disminución en la resistencia del concreto. Para revertir esta situación es recomendable: utilizar látex de caucho en cantidades menores al $8 \%$, asimismo la introducción de nanosílice en la mezcla. 
Para aumentar la resistencia a la tracción indirecta se debe remplazar el $5 \%$ del cemento con metacaolín, pero utilizando geopolímeros tienden a disminuir la resistencia a la tracción indirecta y las cenizas volantes, por encima del $20 \%$, afectan las propiedades mecánicas del concreto permeable.

Unos de los materiales reciclables son las conchas marinas que afectan la resistencia a la compresión del concreto permeable, disminuyendo de $42 \mathrm{MPa}$ a $11 \mathrm{MPa}$; por otro lado, tienen sus ventajas de aumentar la porosidad de $5.2 \%$ a $27.2 \%$.

La utilización de agregado reciclado genera un aumento inicial de la resistencia del concreto permeable en un rango del $15 \%$ al $20 \%$, independientemente del tipo de cemento o agregado empleado en su elaboración.

Para obtener una resistencia superior de $16.4 \%$ a la comprensión, será necesario adicionar un $35 \%$ de caliza comercial molida en el concreto con una relación $\mathrm{A} / \mathrm{C}$ desde 0.44 a 0.49 .

Para finiquitar con la investigación, se concluye que la producción de concreto permeable es una buena opción para el desarrollo de ciclovías, aceras y pavimentos en estas épocas modernas, cuya tendencia es la búsqueda del desarrollo sostenible debido a la utilización de materiales reciclados durante su producción y la disminución en los costos de elaboración.

\section{AGRADECIMIENTOS}

Agradecemos a la Escuela de Ingeniería Civil de la Universidad Señor de Sipán por habernos facilitado el acceso a las bases indexadas para poder hacer la búsqueda de los artículos. Es un proyecto que no está financiado por ninguna institución.

\section{CONFLICTO DE INTERESES}

Los autores declaran no tener conflictos de intereses.

\section{CONTRIBUCIÓN DE LOS AUTORES}

Todos los autores contribuyeron, de igual manera, a la compilación y análisis de la información, así como con la redacción y discusión sobre el tema. Específicamente:

Rosario Dolores Cornejo Ramos concibió la idea del artículo y se centró en la elaboración de los vínculos entre las diferentes estructuras con aspectos de innovación.

Jhon Emanuel Ayala López contribuyó en el diseño de la investigación, desarrollando la argumentación para los filtros de las búsquedas iniciales y técnicas de análisis.

Elmer Gil Ahumada acompañó la argumentación, análisis de los resultados y revisión crítica del artículo.

Sócrates Pedro Muñoz Pérez realizó la validación del procedimiento, el análisis de los resultados y la redacción de la versión final del artículo.

\section{REFERENCIAS}

[1] F. H. M. Ahmad; M. H. Jamal; A. R. M. Sam; N. F. Haron, "Eco-composite Porous Concrete Drainage Systems: An Alternative Mitigation for Urban Flood Management”, en Proceedingsofthe International 
Conferenceon Civil, Offshore and Environmental Engineering, Kuching, 2021, pp. $195-201$. https://doi.org/10.1007/978-981-33-6311-3_23

[2] R. Zhong; Z. Leng; C. Poon, "Research and application of pervious concrete as a sustainable pavement material: A state-of-the-art and state-of-the-practice review", Constr. Build. Mater, vol. 183, pp. 544-553, Sep. 2018. https://doi.org/10.1016/j.conbuildmat.2018.06.131

[3] S. Cabello; L. Campuzano; J. Espinoza; C. Sánchez, "Concreto poroso: Constitución, Variables influyentes y Protocolos para su caracterización”, Cumbres, vol. 1, no. 1, pp. 64-69, Jun. 2015. https://doi.org/10.48190/cumbres.v1n1a9

[4] G. L. Vieira; J. Z. Schiavon; P. M. Borges; S. R. da Silva; J. J. de Oliveira Andrade, "influence of recycled aggregate replacement and fly ash content in performance of pervious concrete mixtures," J. Clean. Prod., vol. 271, p. 122665, Oct. 2020. https://doi.org/10.1016/j.jclepro.2020.122665

[5] G. F. B. Sandoval; I. Galobardes; R. S. Teixeira; B. M. Toralles, "Comparison between the falling head and the constant head permeability tests to assess the permeability coefficient of sustainable Pervious Concretes", Case Stud. Constr. Mater., vol. 7, pp. 317-328, Dec. 2017. https://doi.org/10.1016/j.cscm.2017.09.001

[6] R. Pieralisi; G. F. B. Sandoval; L. Segura-Castillo; M. N. C. Barbosa; S. T. Assunção, "Contribuição Para O Desenvolvimento De Uma Metodologia De Dosagem Para Concreto Permeável Baseada No Desempenho,", J. Urban Technol. Sustain., vol. 3, no. 1, pp. 18-27, Dec. 2020. https://doi.org/10.47842/juts.v3i1.19

[7] A. K. Chandrappa; K. P. Biligiri, "Pervious concrete as a sustainable pavement material - Research findings and future prospects: A state-of-the-art review", Constr. Build. Mater., vol. 111, pp. 262-274, May 2016. https://doi.org/10.1016/j.conbuildmat.2016.02.054

[8] A. Bonicelli; L. R. Pianeta, "Performance and Applications of Pervious Concrete Pavement Material as an Overlay on Existent Concrete Slabs”, IOP Conf. Ser. Mater. Sci. Eng., vol. 471, no. 3, p. 032061, Feb. 2019. https://doi.org/10.1088/1757-899X/471/3/032061

[9] S. Chen; Y. Zhao; Y. Bie, "The prediction analysis of properties of recycled aggregate permeable concrete based on back-propagation neural network", J. Clean. Prod., vol. 276, p. 124187, Dec. 2020. https://doi.org/10.1016/j.jclepro.2020.124187

[10] E. Khankhaje; M. Rafieizonooz; M. R. Salim; J. Mirza, Salmiati; M. W. Hussin, "Comparing the effects of oil palm kernel shell and cockle shell on properties of pervious concrete pavement", Int. J. Pavement Res. Technol., vol. 10, no. 5, pp. 383-392, Sep. 2017. https://doi.org/10.1016/j.ijprt.2017.05.003

[11] Q. Zhang; X. Feng; X. Chen; K. Lu, "Mix design for recycled aggregate pervious concrete based on response surface methodology", Constr. Build. Mater, vol. 259, pp. 1-11, Oct. 2020. https://doi.org/10.1016/j.conbuildmat.2020.119776

[12] D. Zuluaga-Castrillón; J. F. Hernández-Ruiz; F. Vargas-Galvis; M. E. López-Gómez; C. C. PalacioEspinosa, "Dynamic drilling as an alternative method for determining the mechanical behavior of refractories materials", TecnoLógicas, vol. 20, no. 39, pp. 99-114, May 2017. https://doi.org/10.22430/22565337.694

[13] C. A. Martínez; J. F. Díaz; R. Duque, "Formwork Layout for Walls Using Modular Formworks", TecnoLógicas, vol. 22, pp. 1-18, Dic. 2019. https://doi.org/10.22430/22565337.1509

[14] Z. Yu et al., "Performance of permeable pavement systems on stormwater permeability and pollutant removal," Environ. Sci. Pollut. Res., vol. 28, no. 22, pp. 28571-28584, Feb. 2021. https://doi.org/10.1007/s11356-021-12525-5

[15] K. Dall Bello De Souza Risson; G. F. B. Sandoval; F. S. Cofani Pinto; M. Camargo; A. Campos De Moura; B. Martins Toralles, "Molding procedure for pervious concrete specimens by density control", Case Stud. Constr. Mater., vol. 15, p. e00619, Dec. 2021. https://doi.org/10.1016/j.cscm.2021.e00619

[16] R. Pieralisi; S. H. P. Cavalaro; A. Aguado, "Advanced numerical assessment of the permeability of pervious concrete", Cem. Concr. Res., vol. 102, pp. 149-160, Dec. 2017. https://doi.org/10.1016/j.cemconres.2017.09.009

[17] E. Khankhaje et al., "Properties of quiet pervious concrete containing oil palm kernel shell and cockleshell", Appl. Acoust., vol. 122, pp. 113-120, Jul. 2017. https://doi.org/10.1016/j.apacoust.2017.02.014

[18] H. A. Rondón-Quintana; C. F. Urazán-Bonells; S. B. Chaves-Pabón, "Influence of compaction temperature on the resistance of a stabilized granular material with asphalt cement", TecnoLógicas, vol. 18, no. 34, p.p, 51-62, Jan. 2015. https://doi.org/10.22430/22565337.218

[19] S. Zhang; P. He; L. Niu, "Mechanical properties and permeability of fiber-reinforced concrete with recycled aggregate made from waste clay brick", J. Clean. Prod., vol. 268, pp. 121690, Sep. 2020. https://doi.org/10.1016/j.jclepro.2020.121690

[20] B. Liu; J. Shi; M. Sun; Z. He, H. Xu; J. Tan, "Mechanical and permeability properties of polymer-modified concrete using hydrophobic agent”, J. Build. Eng., vol. 31, p. 101337, Sep. 2020. https://doi.org/10.1016/j.jobe.2020.101337 
[21] W. Zhao; Y. Zhang; L. Li, W. Su; G. Ma; B. Li, "Thermal characteristics of porous concrete in a hydronic road heating system", Appl. Therm. Eng., vol. 182, p. 116074, Jan. 2021. https://doi.org/10.1016/j.applthermaleng.2020.116074

[22] R. J. Mikami; P. Kruger; E. Pereira; A. C. B. Kummer; M. M. R. Döll, "Influência do teor de cerâmica vermelha do agregado reciclado nas propriedades do concreto permeável”, Matéria (Rio Janeiro), vol. 23, no. 3, Oct. 2018. https://doi.org/10.1590/S1517-707620180003.0497

[23] Y. Zhang; H. Li; A. Abdelhady; J. Yang; H. Wang, "Effects of specimen shape and size on the permeability and mechanical properties of porous concrete", Constr. Build. Mater., vol. 266, Part B, p. 121074, Jan. 2021.https://doi.org/10.1016/j.conbuildmat.2020.121074

[24] F. Yu; D. Sun; J. Wang; M. Hu, "Influence of aggregate size on compressive strength of pervious concrete", Constr. Build. Mater., vol. 209, pp. 463-475, Jun. 2019. https://doi.org/10.1016/j.conbuildmat.2019.03.140

[25] O. AlShareedah; M. M. Haider; S. Nassiri, "Correlating Laboratory and Field Compaction Levels to Achieve Optimum In Situ Mechanical Properties for Pervious Concrete Pavements”, J. Mater. Civ. Eng., vol. 32, no. 10, p. 04020278, Oct. 2020. https://doi.org/10.1061/(ASCE)MT.1943-5533.0003361

[26] R. Pieralisi; S. H. P. Cavalaro; A. Aguado, "Discrete element modelling of mechanical behaviour of pervious concrete", Cem. Concr. Compos., vol. 119, p. 104005, May 2021. https://www.sciencedirect.com/science/article/pii/S0958946521000743?dgcid=rss_sd_all

[27] B. S. Mohammed; M. S. Liew; W. S. Alaloul; V. C. Khed; C. Y. Hoong; M. Adamu, "Properties of nano-silica modified pervious concrete", Case Stud. Constr. Mater., vol. 8, pp. 409-422, Jun. 2018. https://doi.org/10.1016/j.cscm.2018.03.009

[28] W. M. P. de Araújo; F. P. Avelino; M. de S. Picanço; A. N. Macêdo, "Study of the physical and mechanical properties of permeable concrete with the addition of TiO 2 for the treatment of sewage", Rev. IBRACON Estruturas e Mater., vol. 13, no. 5, p.p. 1-16, Mar. 2020.https://doi.org/10.1590/S1983-41952020000500014

[29] L. M. Tavares; C. S. Kazmierczak, "The influence of recycled concrete aggregates in pervious concrete", Rev. IBRACON Estruturas e Mate., vol. 9, no. 1, pp. 75 - 90, Feb. 2016. https://doi.org/10.1590/S1983$\underline{41952016000100006}$

[30] B. Jain; V. Jain; G. Sancheti, "A study on workability of silica fume and iron dust added concrete", IOP Conf. Ser. Earth Environ. Sci., vol. 796, no. 1, p. 012072, Aug. 2021. https://doi.org/10.1088/17551315/796/1/012072

[31] Y. Tan; Y. Zhu; H. Xiao, "Evaluation of the Hydraulic, Physical, and Mechanical Properties of Pervious Concrete Using Iron Tailings as Coarse Aggregates", Appl. Sci., vol. 10, no. 8, p. 2691, Apr. 2020. https://doi.org/10.3390/app10082691

[32] J. Chen; R. Chu; H. Wang; L. Zhang; X. Chen; Y. Du, "Alleviating urban heat island effect using highconductivity permeable concrete pavement”, J. Clean. Prod., vol. 237, p. 117722, Nov. 2019. https://doi.org/10.1016/j.jclepro.2019.117722

[33] D. Singh; S. P. Singh, "Influence of recycled concrete aggregates and blended cements on the mechanical properties of pervious concrete", Innov. Infrastruct. Solut., vol. 5, no. 3, p. 66, Dec. 2020. https://doi.org/10.1007/s41062-020-00314-x

[34] X. Cai; K. Wu; W. Huang; J. Yu; H. Yu, "Application of recycled concrete aggregates and crushed bricks on permeable concrete road base", Road Mater. Pavement Des., vol. 22, no. 10, pp. 2181-2196, Oct. 2021. https://doi.org/10.1080/14680629.2020.1742193

[35] X. Yao; H. Liao; H. Dong; F. Yang; Y. Yao; W. Wang, "Influence of water repellent on the property of solid waste based sulfoaluminate cement paste and its application in lightweight porous concrete", Constr. Build. Mater., vol. 282, pp. 122731, May 2021. https://doi.org/10.1016/j.conbuildmat.2021.122731

[36] E. Güneyisi; M. Gesoğlu; Q. Kareem; S. İpek, "Effect of different substitution of natural aggregate by recycled aggregate on performance characteristics of pervious concrete", Mater. Struct., vol. 49, no. 1-2, pp. 521-536, Jan. 2016. https://doi.org/10.1617/s11527-014-0517-y

[37] F. Wen; K. Zhang; H. Fan; S. Zhai; F. Liu, "Physical Properties and Seepage Characteristics of Optimized Fiber-Reinforced Permeable Concrete”, J. Mater. Civ. Eng., vol. 33, no. 5, p. 04021093, May 2021. https://doi.org/10.1061/(ASCE)MT.1943-5533.0003590

[38] H. Wu; Z. Liu; B. Sun; J. Yin, "Experimental investigation on freeze-thaw durability of Portland cement pervious concrete (PCPC)”, Constr. Build. Mater., vol. 117, pp. 63-71, Aug. 2016. https://doi.org/10.1016/j.conbuildmat.2016.04.130

[39] F. Yu; D. Sun; G. Sun; S. Ling; M. Hu; J. Ma, "A modified mix design method for pervious concrete based on Mohr-Coulomb failure criterion", Constr. Build. Mater., vol. 269, pp. 121801, Feb. 2021. https://doi.org/10.1016/j.conbuildmat.2020.121801

[40] A. Schackow; C. Effting; V. G. Barros; I. R. Gomes; V. S. da Costa Neto; M. S. Delandréa, "Permeable concrete plates with wastes from the paper industry: Reduction of surface flow and possible applications", Constr. Build. Mater., vol. 250, p. 118896, Jul. 2020. https://doi.org/10.1016/j.conbuildmat.2020.118896 
[41] M. Pereira; J. Carbajo; L. Godinho; J. Ramis; P. Amado-Mendes, "Improving the sound absorption behaviour of porous concrete using embedded resonant structures”, J. Build. Eng., vol. 35, p. 102015, Mar. 2021. https://doi.org/10.1016/j.jobe.2020.102015

[42] Z. Wang; D. Zou; T. Liu; A. Zhou; M. Shen, "A novel method to predict the mesostructure and performance of pervious concrete", Constr. Build. Mater., vol. 263, p. 120117, Dec. 2020. https://doi.org/10.1016/j.conbuildmat.2020.120117

[43] E. J. Elizondo-Martinez; V. C. Andrés-Valeri; J. Rodriguez-Hernandez; D. Castro-Fresno, "A New Design Methodology for Improving Porous Concrete Properties to Achieve Multifunctional and Sustainable Pavements", En Proceedings of the 5th International Symposium on Asphalt Pavements \& Environment (APE). Switzerland: Springer, 2020, pp. 491-499. https://link.springer.com/content/pdf/10.1007\%2F978-3030-29779-4.pdf

[44] G. F. B. Sandoval; I. Galobardes; A. C. De Moura; B. M. Toralles, "Hydraulic behavior variation of pervious concrete due to clogging", Case Stud. Constr. Mater., vol. 13, p. e00354, Dec. 2020. https://doi.org/10.1016/j.cscm.2020.e00354

[45] E. J. Elizondo-Martínez; V. C. Andrés-Valeri; L. Juli-Gándara; J. Rodriguez-Hernandez, "Multi-criteria optimum mixture design of porous concrete pavement surface layers", Int. J. Pavement Eng., pp. 1-10, May 2020. https://www.tandfonline.com/doi/epub/10.1080/10298436.2020.1768254?needAccess=true

[46] A. K. Chandrappa; K. P. Biligiri, "Comprehensive investigation of permeability characteristics of pervious concrete: A hydrodynamic approach," Constr. Build. Mater., vol. 123, pp. 627-637, Oct. 2016. https://doi.org/10.1016/j.conbuildmat.2016.07.035

[47] Y. Zaetang; V. Sata; A. Wongsa; P. Chindaprasirt, "Properties of pervious concrete containing recycled concrete block aggregate and recycled concrete aggregate", Constr. Build. Mater., vol. 111, pp. 15-21, May 2016. https://doi.org/10.1016/j.conbuildmat.2016.02.060

[48] T. M. Borhan; R. J. Al Karawi, "Experimental investigations on polymer modified pervious concrete", Case Stud. Constr. Mater., vol. 12, p. e00335, Jun. 2020. https://doi.org/10.1016/j.cscm.2020.e00335

[49] O. AlShareedah; S. Nassiri; Z. Chen; K. Englund; H. Li; O. Fakron, "Field performance evaluation of pervious concrete pavement reinforced with novel discrete reinforcement", Case Stud. Constr. Mater., vol. 10, p. e00231, Jun. 2019. https://doi.org/10.1016/j.cscm.2019.e00231

[50] D. H. Nguyen; M. Boutouil; N. Sebaibi; F. Baraud; L. Leleyter, "Durability of pervious concrete using crushed seashells", Constr. Build. Mater., vol. 135, pp. 137-150, Mar. 2017. https://doi.org/10.1016/j.conbuildmat.2016.12.219

[51] J. Liu; Y. Li , "Runoff purification effects of permeable concrete modified by diatomite and zeolite powder", Advances in Materials Science and Engineering, vol. 2020, pp. 1-11, Ago. 2020. https://doi.org/10.1155/2020/1081346

[52] C. Gaedicke; A. Marines; L. Mata; F. Miankodila, "Efecto del uso de materiales reciclados y métodos de compactación en las propiedades mecánicas e índice de reflectancia solar del hormigón permeable”, Rev. Ing. construcción, vol. 30, no. 3, pp. 159-167, Dec. 2015. http://dx.doi.org/10.4067/S071850732015000300001

[53] M. R. Pinto; C. Carrasco; K. Caballero, "Estudio experimental del concreto poroso con la incorporación de distintas granulometrías", I+D Tecnológico, vol. 14, no. 2, pp. 57-65, Dec. 2018. https://doi.org/10.33412/idt.v14.2.2074

[54] R. J. Mikami; A. C. B. Kummer; M. M. R. Döll, "Leaching of Pervious Concrete Produced Using Mixed Recycled Aggregates”, Brazilian Arch. Biol. Technol., vol. 63, 2020. https://doi.org/10.1590/1678-4324$\underline{2020180408}$

[55] B. M. Taheri; A. M. Ramezanianpour; S. Sabokpa; M. Gapele, "Experimental evaluation of freeze-thaw durability of pervious concrete", J. Build. Eng., vol. 33, p. 101617, Jan. 2021. https://doi.org/10.1016/j.jobe.2020.101617

[56] G. F. Barreto Sandoval; I. Galobardes Reyes; N. Schwantes-Cezario; A. Campos Moura; B. Martins Toralles, "Correlation between Permeability and Porosity for Pervious Concrete (PC)", DYNA, vol. 86, no. 209, pp. 151-159, Apr. 2019. https://doi.org/10.15446/dyna.v86n209.77613

[57] V. A. Ulloa-Mayorga; M. A. Uribe-Garcés; D. P. Paz-Gómez; Y. A. Alvarado; B. Torres; I. Gasch, "Performance of pervious concrete containing combined recycled aggregates", Ing. e Investig., vol. 38, no. 2, pp. 34-41, Aug 2018. http://dx.doi.org/10.15446/ing.investig.v38n2.67491

[58] A. F. H. Sherwani; R. Faraj; K. H. Younis; A. Daraei,, "Strength, abrasion resistance and permeability of artificial fly-ash aggregate pervious concrete", Case Stud. Constr. Mater., vol. 14, pp. 1-15, Jun. 2021. https://doi.org/10.1016/j.cscm.2021.e00502

[59] E.-J. Elizondo-Martinez; P. Tataranni; J. Rodriguez-Hernandez; D. Castro-Fresno, "Physical and Mechanical Characterization of Sustainable and Innovative Porous Concrete for Urban Pavements Containing Metakaolin", Sustainability, vol. 12, no. 10, p. 4243, May 2020. https://doi.org/10.3390/su12104243 
[60] G. H. Faisal; A. J. Jaeel; A. L. Gasham, "BOD and COD reduction using porous concrete pavements", Case Studies in Construction Materials, vol. 13, pp. 1-16, Dic. 2020. https://doi.org/10.1016/j.cscm.2020.e00396

[61] J. Zhao et al., "Comparison of Field Infiltration Test Methods for Permeable Pavement: Towards an Easy and Accurate Method", CLEAN - Soil, Air, Water, vol. 47, no. 8, p. 1900174, Aug. 2019. https://doi.org/10.1002/clen.201900174

[62] M. N. Marinho; A. P. Coutinho; S. M. dos Santos Neto; C. A. Casagrande; G. T. L. Santos; A. M. P. Carneiro, "Mathematical modeling of the infiltration in a permeable pavement on the field scale", $R B R H$, vol. 25, 2020. https://doi.org/10.1590/2318-0331.252020200052

[63] E. Lee; J. Ahn; H.-S. Shin, "Evaluation of surface infiltration rate of permeable block pavements using single ring infiltrometer", Desalin. WATER Treat., vol. 104, pp. 286-293, 2018. https://doi.org/10.5004/dwt.2018.21818

[64] B. W. Ono; J. T. Balbo; A. Cargnin, "Análise da capacidade de infiltração em pavimento permeável de bloco de concreto unidirecionalmente articulado", Transportes, vol. 25, no. 3, p. 90-101, Oct. 2017. https://doi.org/10.14295/transportes.v25i3.1314

[65] W. D. Martin; B. J. Putman, "Comparison of methods for measuring porosity of porous paving mixtures", Constr. Build. Mater., vol. 125, pp. 299-305, Oct. 2016. https://doi.org/10.1016/j.conbuildmat.2016.08.038

[66] N. A. Brake; H. Allahdadi; F. Adam, "Flexural strength and fracture size effects of pervious concrete", Constr. Build. Mater., vol. 113, pp. 536-543, Jun. 2016. https://doi.org/10.1016/j.conbuildmat.2016.03.045

[67] A. Torres; J. Hu; A. Ramos, "The effect of the cementitious paste thickness on the performance of pervious concrete", Constr. Build. Mater., vol. 95, pp. 850-859, Oct. 2015. https://doi.org/10.1016/j.conbuildmat.2015.07.187

[68] K. S. B. Prasad; S. Lahari Anisha; N. Pavan Kumar, "Experiment on Mechanical Properties of Pervious Concrete", Int. J. Recent Technol. Eng., vol. 8, no. 2S8, pp. 1004-1007, Aug. 2019. https://doi.org/10.35940/ijrte.B1192.0882S819

[69] R. Pieralisi; S. H. P. Cavalaro; A. Aguado, "Discrete element modelling of the fresh state behavior of pervious concrete", Cem. Concr. Res., vol. 90, pp. 6-18, Dec. 2016. https://doi.org/10.1016/j.cemconres.2016.09.010

[70] F. B. Pereira da Costa; L. M. Haselbach; L. C. P. da Silva Filho, "Pervious concrete for desired porosity: Influence of w/c ratio and a rheology-modifying admixture", Constr. Build. Mater, vol. 268, pp. 1-12, 25 Jan. 2021. https://doi.org/10.1016/j.conbuildmat.2020.121084

[71] S. T. Martins Filho; E. M. Bosquesi; J. R. Fabro; R. Pieralisi, "Characterization of pervious concrete focusing on non-destructive testing", Rev. IBRACON Estruturas e Mater., vol. 13, no. 3, p. 483 - 500, Jun. 2020. https://doi.org/10.1590/S1983-41952020000300003

[72] M. Kovác; A. Sicáková, "Pervious Concrete as an Environmental Solution for Pavements: Focus on Key Properties", Environments, vol. 5, no. 1, pp. 1-9, Jan. 2018. https://doi.org/10.3390/environments5010011

[73] C.-W. Tang; C.-K. Cheng; C.-Y. Tsai, "Mix Design and Mechanical Properties of High-Performance Pervious Concrete", Materials, vol. 12, no. 16, p. 2577, Aug. 2019. https://doi.org/10.3390/ma12162577

[74] P. R. Guerra Chayña; C. E. Guerra Ramos, "Design of a rigid permeable pavement as a sustainable urban drainage system", Fides Et Ratio, vol. 20, no. 20, pp. 121-140, Sep. 2020. http://www.scielo.org.bo/scielo.php?script=sci_arttext\&pid=S2071-081X2020000200008\&lng=es\&tlng=

[75] R. R. Singh; A. J. S. Sidhu, "Fracture and fatigue study of pervious concrete with 15-20\% void ratio", Sādhanā, vol. 45, no. 1, p. 151, Jun. 2020. https://doi.org/10.1007/s12046-020-01374-6

[76] Z. Yan; L. Li; M. Chen; L. Lu; P. Zhao; X. Cheng, "The rheology of a cement paste and the frost resistance of a permeable concrete with an emulsified asphalt modified by a silane coupling agent", Ceramics Silikáty, vol. 64, no. 2, pp. 125-134, Jan. 2020. https://doi.org/10.13168/cs.2020.0001

[77] S. P. Yap; P. Z. C. Chen; Y. Goh; H. A. Ibrahim; K. H. Mo; C. W. Yuen, "Characterization of pervious concrete with blended natural aggregate and recycled concrete aggregates", J. Clean. Prod., vol. 181, pp. 155-165, Apr. 2018. https://doi.org/10.1016/j.jclepro.2018.01.205

[78] J. J. Randrianarimanana, N. Sebaibi y M. Boutouil, "Laboratory Analysis of Stormwater Runoff Hydraulic and Pollutant Removal Performance of Pervious Concrete Based on Seashell By-Products", Int. J. Civil, Environ. Struct. Constr. Archit. Eng., vol. 11, no. 8, pp. 1059-1068, Ago. 2017. https://www.researchgate.net/publication/319329224_Laboratory_Analysis_of_Stormwater_Runoff_Hydr aulic_and_Pollutant_Removal_Performance_of_Pervious_Concrete_Based_on_Seashell_By-Products

[79] J. L. Z. Tarqui; M. de F. Carvalho; C. M. L. dos Santos; J. E. dos Santos, "Avaliação do escoamento superficial de águas pluviais em pavimento de blocos de solo-cimento", Eng. Sanit. e Ambient., vol. 24, no. 2, pp. 403-410, Apr. 2019. https://doi.org/10.1590/S1413-41522019153148

[80] Y.-Y. Cheng; S.-L. Lo; C.-C. Ho; J.-Y. Lin; S. L. Yu, "Field Testing of Porous Pavement Performance on Runoff and Temperature Control in Taipei City", Water, vol. 11, no. 12, p. 2635, Dec. 2019. https://doi.org/10.3390/w11122635 
[81] L. Moretti; P. Di Mascio; C. Fusco, "Porous Concrete for Pedestrian Pavements", Water, vol. 11, no. 10, p. 2105, Oct. 2019. https://doi.org/10.3390/w11102105

[82] N. Xie; M. Akin; X. Shi, "Permeable concrete pavements: A review of environmental benefits and durability", J. Clean. Prod., vol. 210, pp. 1605-1621, Feb. 2019. https://doi.org/10.1016/j.jclepro.2018.11.134

[83] X. Chen; H. Wang; H. Najm, G. Venkiteela; J. Hencken, "Evaluating engineering properties and environmental impact of pervious concrete with fly ash and slag", J. Clean. Prod., vol. 237, p. 117714, Nov. 2019. https://doi.org/10.1016/j.jclepro.2019.117714 